\title{
Human Tracking Using Near Field Imaging
}

\author{
Rimminen Henry, Linnavuo Matti, Sepponen Raimo \\ Applied electronics laboratory \\ Helsinki University of Technology \\ Espoo, Finland \\ matti.linnavuo@tkk.fi
}

\begin{abstract}
The sensor system presented in this paper utilizes near field imaging for wide area human location and tracking. The sensor system provides reliable location data of humans by measuring slight impedance changes between elements of a thick film sensor matrix under the floor covering. The sensor is completely rigid and undetectable. The low cost and scalable design allows the system to be spread across the whole indoor surface area. The system aims to ensure the efficiency of the elderly care system. Ageing of the population structure in Finland generates an eminent need for more resources in this field. The main pilot sites of the described system are in the senior homes, where the personnel will be most stressed in the near future. The location data can be used for various purposes such as making alarms of senior persons getting up from bed during the night risking them selves of losing balance. Making an alarm during falling down on the floor has an obvious benefit for the habitants of old age homes.
\end{abstract}

Keywords: Near field imaging, Human tracking system

\section{INTRODUCTION}

Ageing of the Finnish population structure generates an eminent need for more resources in elderly care. Various technologies are utilized to assist the caretakers. Many pervasive applications, especially technologies to support ambient assisted living require methods of tracking people. The tracking function can be realized in various ways depending on the application, required area and resolution.[1] In principle, two methods are available: the active method, where the person is tagged with a transponder [2] and passive method which observes the person proper [3]. When pervasive systems grow more prevalent, cost effectiveness will be an issue. Thus innovative in vivo solutions are needed instead of research grade in vitro realizations. In elderly care, active methods are unacceptable because of significant maintenance and attention requirement of the transponder.[4] There are many challenges on pervasive computing systems, one of which is the requirement of invisibility [4]. This requirement is best fulfilled by sensors embedded in walls or floor. Our system relies on passive method with sensors located under floor surface. There are many floor based location systems which measure the static [5], [6] and dynamic [7] weight distribution. Static realizations, however, are complex and inaccurate. The human body has a relatively small impedance and thus it is suitable to be sensed by the electric field. The

Permission to make digital or hard copies of all or part of this work for personal or classroom use is granted without fee provided that copies are not made or distributed for profit or commercial advantage and that copies bear this notice and the full citation on the first page. To copy otherwise, to republish, to post on servers or to redistribute to lists, requires prior specific permission and/or a fee. PERVASIVEHEALTH 2008, 30 Jan - 1 Feb. Tampere, Finland

Copyright $@ 2008$ ICST 978-963-9799-15-8

DOI 10.4108/ICST.PERVASIVEHEALTH2008.2533 applications so far are limited to special biopotential measurements [8], [9] with some exceptions in computer or other electronic appliances interfaces [10]. We have applied the electric field sensing to scalable, invisible and low cost sensor and method for human tracking.

\section{MAterials And Methods}

\section{A. Measurement Principle}

The measurement principle is in Fig. 1. The measurement sensor consists of a metal thick film squares measuring approx. $300 \times 300 \mathrm{~mm}$ with $400 \mathrm{~mm}$ pitch. Sensors are protected by plastic laminate. The total thickness of the multilayer sensor is $160 \mu \mathrm{m}$. The design of sensor and electronics allow applications on floors of $10 \mathrm{~m}$ in width and arbitrary length. A low frequency low voltage carrier signal is fed to a single sensor element (primary element) while the others (secondary elements) are grounded providing a return path for the signal. An impedance is composed of the electric field coupling between the primary and secondary elements to the conductive object, the resistive properties of the conductive object, and the inductance of all conductors. Any conductive object appearing on top of the sensor array will affect the impedance and current flow between the primary and secondary sensor elements. Thus the system measures impedance variations at a point frequency. Every element in the thick film sensor matrix is then scanned sequentially by multiplexing the carrier signal. To track human beings successfully, one needs multiple updates per second.

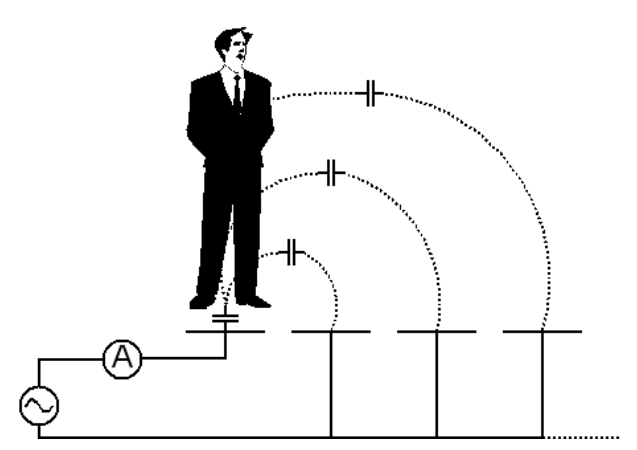

Figure 1 Measurement principle. Alternating current is fed to a single sensor plate and current flow is measured. The whole sensor element matrix is scanned sequentially. Conductive objects such as humans affect the current flow. Common floor coverings do not disturb the measurement, because they are usually dielectric. 


\section{B. Verification Methods}

To verify the functionality of the measurement system three tests were performed. The most obvious parameter would be the sensitivity of the system. By sensitivity we refer to the systems response to impedance change stimulus. To measure the sensitivity, a set of ceramic capacitors were connected between two randomly selected neighboring sensor elements. The connection was made by soldering a two terminal socket on the element leads to ensure proper contact with the capacitor leads. The values of the capacitors were chosen between 1 and 10 picofarads to achieve an absolute impedance change scale from 2 to 20 ohms. These capacitors were then plugged in sequentially during normal operation and the corresponding results from the analog-to-digital converter (ADC) were written down. To ensure minimal error in the actual measurement, the capacitance value of each ceramic capacitor was first verified with a commercial LCR-meter (4263A, Hewlett Packard, USA) at a $100 \mathrm{kHz}$ measurement frequency.

The second most obvious parameter would be the signalto-noise ratio (SNR) of the whole system. To accurately measure a practical SNR, a human phantom was constructed. See Fig. 2. The purpose was to couple two neighboring sensor elements together electrically. This kind of leg to leg coupling has proven to be the dominant coupling mode in the case of humans walking on the sensors. The legs were constructed of steel and feet were made of aluminum. Both legs were isolated from each other and mounted on a dielectric framework constructed of wood. The frame has no screws or nails on the bottom half. The foot size in European units was chosen to be 41. To determine the ballpark of the needed leg to leg impedance at $100 \mathrm{kHz}$, a series of measurements among the laboratory staff were made. Four people were asked to step on two separate copper plates with bare feet. The leg to leg impedance was then measured at a $100 \mathrm{kHz}$ measurement frequency with the same LCR-meter mentioned earlier. Results can be seen in Table 1. An absolute impedance value of approximately $500 \mathrm{ohms}$ was calculated to be the average of four people. Thus a $500 \mathrm{ohm}$ resistance was connected between the conductive legs.

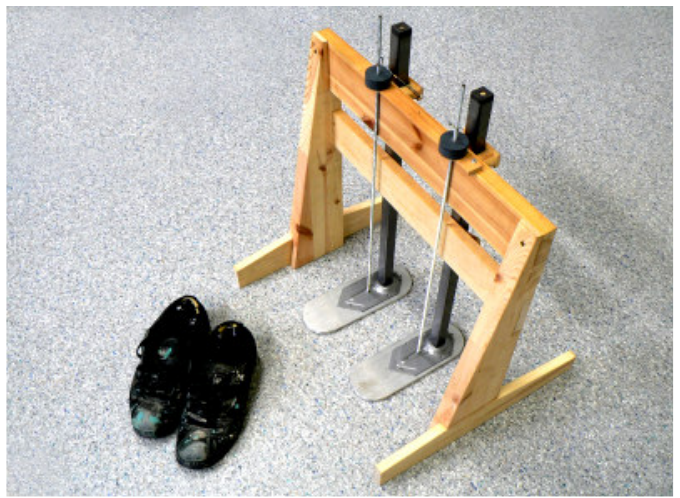

Figure 2 The Human body phantom with adjustable legs. European foot size is 41. Leg to leg Impedance is $500 \Omega$. Test shoes used in the tests had $15 \mathrm{~mm}$ thick synthetic rubber soles. Sensor array is under a plastic floor covering.
TABLE I. $\quad$ LEG TO LEG IMPEDANCE

\begin{tabular}{|l|c|c|}
\hline \multirow{2}{*}{ Test subjects } & \multicolumn{2}{|c|}{ Subject properties } \\
\cline { 2 - 3 } & |Impedance| [Q] & Gender \\
\hline Subject 1 & 490 & Male \\
\hline Subject 2 & 460 & Male \\
\hline Subject 3 & 475 & Female \\
\hline Subject 4 & 570 & Male \\
\hline Average & 499 & \\
\hline
\end{tabular}

The third verification method was a simple walking test on the sensor matrix. The purpose was to visually observe the location data. The test subject also intentionally fell down on the floor to demonstrate a fallen pattern. The test subject was a male weighing 85 kilograms. He was wearing synthetic rubber soled sneakers and his walking speed was swift.

\section{RESULTS}

\section{A. System Description}

Several rounds of prototyping were performed by the authors and the following measurement configuration was proven to be an optimum: The current flowing through the primary sensor element is measured by shunt impedance (see Fig. 3). The signal is then amplified with a difference amplifier and mixed with the original carrier using a balanced demodulator. This procedure leaves us with a result, which only has components with a correct frequency and phase. After a low-pass filter we have a dc voltage that has a linear correspondence to the impedance variations. See Fig. 5.

Impedance changes caused by the presence of a human body are approximately one percent of the bulk impedance. The bulk impedance comprises mainly of the stray capacitance of the sensor element leads and the multiplexer units. The static bulk impedance causes a relatively large dc bias voltage which is canceled at the ADC. Both power and communication circuitry are isolated from the sensor electronics.

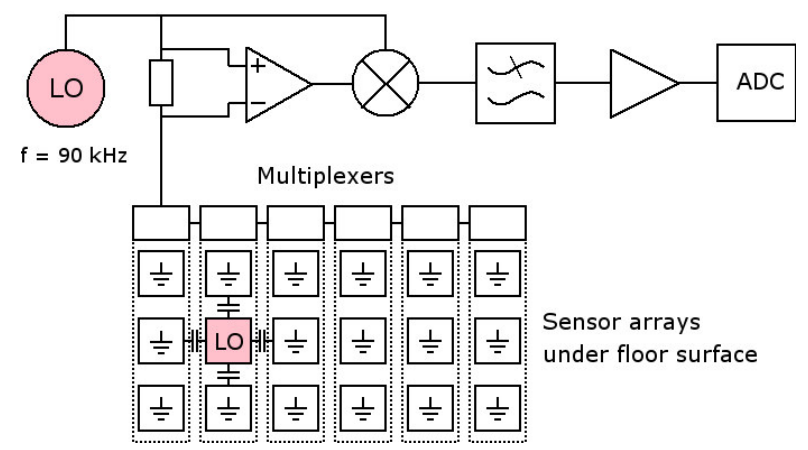

Figure 3 Block diagram of the near field imaging measurement system. The phase sensitive detector takes care of EMI rejection. 


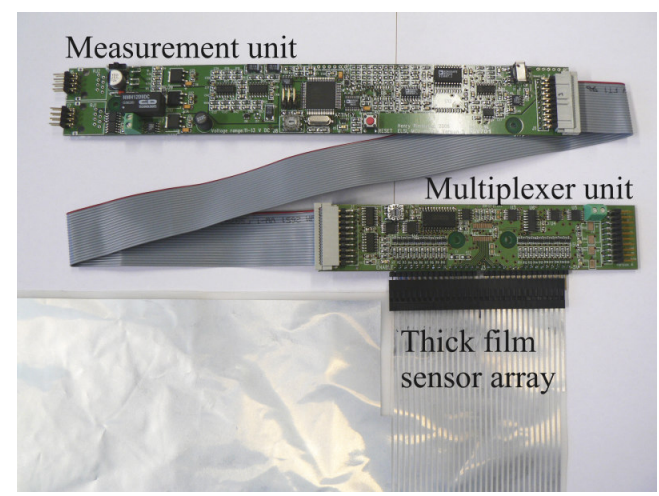

Figure 4 Realization of the circuit boards. One measurement unit is shown here together with the first multiplexer unit, which is connected to a thick film sensor array.

The actual realization of the circuit boards is presented in Fig. 4. Multiplexer units are chained together with the measurement unit using a standard 20-lead flat cable. The shape of the circuit boards is oblong because they are designed to fit in to hollow baseboards along the walls. CAN-bus was chosen to handle the data transfer between the measurement units and the central guidance computer because of its flexibility [11] and immunity to EMI.

The CAN network may consist of 16 measurement units. A measurement unit can manage up to 16 multiplexer units, which are each capable of measuring 20 sensor elements in the sensor arrays. Thus the total number of sensor elements in a single network could be 5120 . Considering that the area of a single sensor element is $0.1 \mathrm{~m}^{2}$, the total area of a single CAN network can be as large as $512 \mathrm{~m}^{2}$.

\section{B. Verification Results}

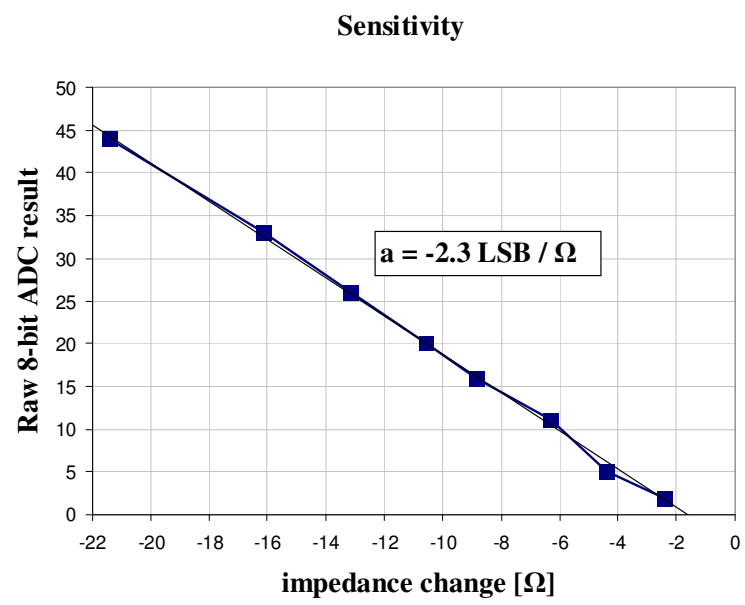

Figure 5 The sensitivity and linearity of the system. The angular coefficient a represents the sensitivity in respect to changes in impedance.

According to the test using ceramic capacitors, the sensitivity of the system is -2.3 least significant bits (LSB) per ohm. See Fig. 5. The smallest change that could be detected persistently during normal scanning was $2.4 \mathrm{ohms}$. This is a
$1.3 \%$ change considering that the bulk impedance between the two sensor elements was 1905 ohms. According to Fig. 5, the linearity error in respect to impedance change is no more than one LSB.

The human body phantom described in the Materials and methods section was used to determine the practical SNR of the system. Four samples were taken using the human phantom. During the tests it was wearing synthetic rubber soled sneakers with approximately 15 millimeter thickness. The measurement unit was set to a continuous sampling mode and the phantom was placed on an optimal location on the sensor with legs adjusted up at a height of 40 centimeters. By optimal location we mean that each foot was centered in the middle of two neighboring sensor elements. Then the legs were pushed down on the floor. The RMS-noise can be measured from the sample when the legs are up and the signal amplitude can be calculated from the step in the signal caused by the legs falling down. Dividing the step amplitude with the RMS-noise value, we have a SNR-value. Taking an average of four samples gives us a good estimate of the actual value. Fig. 6 shows four phantom response samples. The y-axis comprises of 8-bit ADC results converted into impedance. Each of the samples contains 1024 sample points from a measurement period of 12.8 seconds. The actual sampling time is 2.66 milliseconds per sample point, which corresponds to a scanning speed of 376 elements per second during normal operation.

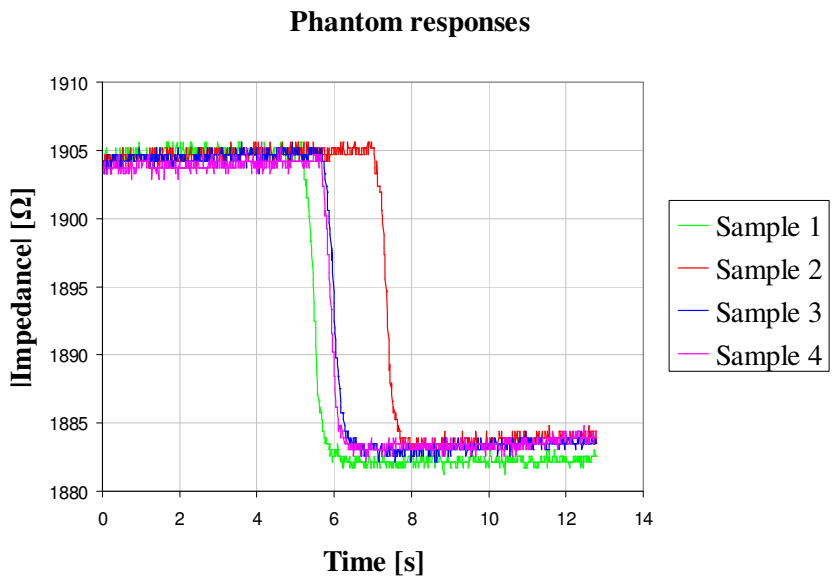

Figure 6 Four responses to the human body phantom stimulus. RMS-noise values were calculated from the first 100 sample points (first 1.25 seconds).

TABLE II. SNR-VALUES

\begin{tabular}{|c|c|c|c|c|}
\hline $\begin{array}{c}\text { Sample } \\
\text { number }\end{array}$ & $\begin{array}{c}\text { Step amplitude } \\
{[\boldsymbol{\Omega}]}\end{array}$ & $\begin{array}{c}\boldsymbol{R M S}-\text { noise } \\
{[\boldsymbol{\Omega}]}\end{array}$ & $\mathbf{S N R}$ & $\begin{array}{c}\text { SNR } \\
{[\boldsymbol{d B}]}\end{array}$ \\
\hline Sample 1 & 23.13 & 0.297 & 77.9 & 37.8 \\
\hline Sample 2 & 21.68 & 0.311 & 69.8 & 36.9 \\
\hline Sample 3 & 21.94 & 0.309 & 71.0 & 37.0 \\
\hline Sample 4 & 21.28 & 0.312 & 68.2 & 36.7 \\
\hline Average & 22.00 & 0.307 & 71.7 & 37.1 \\
\hline
\end{tabular}




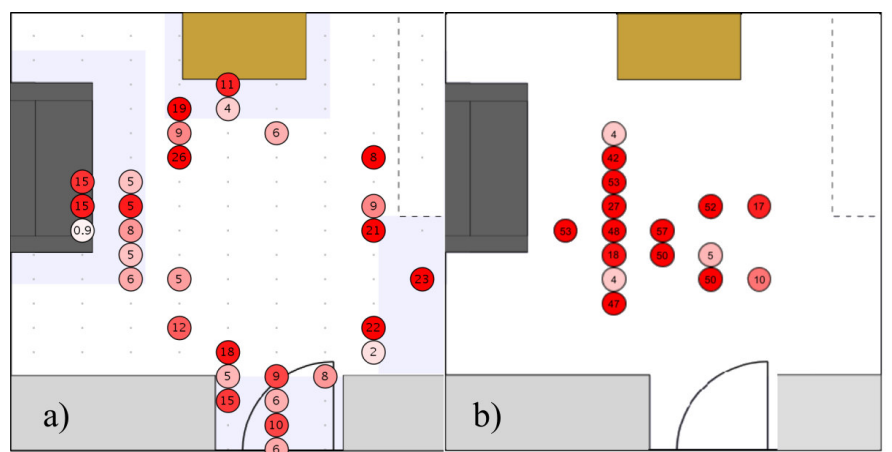

Figure 7. a) Person walking in a test room equipped with the near field imaging floor sensor. Red circles show the detected activity, and the numbers in the red circles represent the impedance changes in ohms. b) Person has fallen on the floor. Test subject is lying on his back, arms spread wide. Head is facing to the left. Resolution here is $450 \mathrm{~mm} \times 250 \mathrm{~mm}$.

Table 2 shows the results of the SNR-calculations. RMSnoise values were calculated using the first 100 sample points. Step amplitudes were calculated by first filtering the samples with a 32 point moving average function, which cancels the short term ripple. After this the step amplitudes were calculated by taking the difference of the highest and lowest sample point between 4 and 10 seconds. The noise value and the step amplitude have been converted into ohms. According to table 2, the system has a 37 decibel SNR using a human body phantom described earlier. The step amplitudes correspond approximately to $22 \mathrm{ohm}$ changes $(1.1 \%)$ in the measured impedance.

Fig. 7, part a) shows the results of a simple walking experiment in a test setup room. The test subject first came in from the door and sat on the sofa one the left. Then he proceeded to the desk and left the room along the wall on the right. Fig 7, part b) shows a fallen pattern where the test subject is lying on his back, arms spread wide.

\section{DISCUSSION}

We have seen that our method and system have some useful properties, such as

- Direct human detection without special tags.

- Thin, solid and stabile sensor design is easy to apply into the building process.

- Various materials can be utilized for floor covering.

- Sensor technology easily adaptable for mass production.

- High SNR and linearity. Adequate scanning speed for small and medium size rooms.

- The ubiquitous and scalable design is an ideal choice for large public or private premises.

Location data of human movement in laboratory conditions have proven to be reliable, but to verify the actual usability there are ongoing pilot installations in several senior houses, largest one having over $800 \mathrm{~m}^{2}$ of monitored space.
The system is suitable for an aid in the elderly care system. The location data are used for various purposes such as making alarms of senior persons getting up from bed during the night risking them selves of losing balance. Making an alarm during falling down on the floor has an obvious benefit for the habitants of old age homes.

Although the first applications of the method and system presented are in elderly care, a variety of human location and tracking needs in building automation and security systems; ergonomic, usability and marketing research could be covered.

\section{ACKNOWLEDGMENT}

This study was supported by European Union and Jenny and Antti Wihuri Foundation. The authors are also grateful for UPM Corporate Venturing for providing the necessary multi layer thick film sensor laminates. Special thanks belong to Kimmo Rajala for constructing the human body phantom and to Juha Lindström for developing the test software.

\section{REFERENCES}

[1] J. Hightower, G. Borriello., "A Survey and Taxonomy of Location Systems for Ubiquitous Computing", Extended paper from Computer, 34(8) p57-66, August 2001.

[2] R. Want, A. Hopper, V. Falcão, J. Gibbons, "The Active Badge Location System", ACM Transactions on Information Systems, vol. 10, pp. 91-102, Jan. 1992.

[3] J, Krumm, S. Harris, B. Meyers, B. Brumitt, M. Hale, S. Shafer, "MultiCamera Multi-Person Tracking for EasyLiving", Proceedings of the Third IEEE International Workshop on Visual Surveillance (VS'2000), 2000.

[4] Y. Kaddoura, J. King, A. Helal, "Cost-Precision Tradeoffs in Unencumbered Floor-Based Indoor Location Tracking," Proceedings of the third International Conference On Smart homes and health Telematic (ICOST), Sherbrooke, Québec, Canada, July 2005.

[5] M. D. Addlesee, A. Jones, F. Livesey and F. Samaria. "The ORL Active Floor”, IEEE Personal Communications, 5(4), 1997, 35-41.

[6] Takuya Murakita, Tetsushi Ikeda, and Hiroshi Ishiguro. Human Tracking using Floor Sensors based on the Markov Chain Monte Carlo Method. Department of Adaptive Machine Systems, Osaka University. Proceedings of the 17th International Conference on Pattern Recognition, 2004.

[7] Lekkala J., Paajanen M., EMFi -New Electret material for sensors and Actuators. Proceedings of the IEEE. 10th International Symposium on Electrets, 1999, $743-746$.

[8] C. J. Harland, T. D. Clark and R. J. Prance, "Electric potential probes New Directions in The Remote Sensing of The Human Body", Measurement Science and Technology, Volume 13, Issue 2, pp. 163169, December, 2001.

[9] W. J. Smith, J. R. LaCourse, "Non-Contact Biopotential Measurement from the Human Body Using a Low-Impedance Charge Amplifier", Bioengineering Conference Proceedings of the IEEE 30th Annual Northeast, pp. 31-32, April, 2004.

[10] T. Zimmerman, J. Smith, J. Paradiso, D. Allport, N. Gershenfeld, "Applying electric field sensing to human-computer interfaces", Proceedings of the SIGCHI conference on Human factors in computing systems, Denver, pp. 280 - 287, 1995

[11] CAN Specification. Version 2.0. Stuttgart, Deutschland: Robert Bosch $\mathrm{GmbH}, 1991.73 \mathrm{p}$. 